\title{
Research on the Route Pricing Optimization Model of the Car-Free Carrier Platform Based on the BP Neural Network Algorithm
}

\author{
Yu-Ang Du \\ School of Statistics and Applied Mathematics, Anhui University of Finance \& Economics, Bengbu 233030, China \\ Correspondence should be addressed to Yu-Ang Du; dyadya200003152015@163.com
}

Received 13 May 2021; Accepted 16 June 2021; Published 23 June 2021

Academic Editor: Huihua Chen

Copyright @ $2021 \mathrm{Yu}$-Ang Du. This is an open access article distributed under the Creative Commons Attribution License, which permits unrestricted use, distribution, and reproduction in any medium, provided the original work is properly cited.

\begin{abstract}
The car-free carrier platform is a product of the rapid development of the modern logistics industry and has a vital strategic value for promoting the construction of a country's comprehensive transportation. However, due to the unreasonable platform pricing model, the industry is currently in a bottleneck period. In order to solve this problem, we established a gray correlation model to calculate the degree of correlation between each characteristic index and platform pricing based on the massive historical transaction data of a certain platform and performed $K$-means clustering on the results to discover the main factors affecting platform pricing. Based on the abovementioned results, we created a pricing optimization model based on the BP neural network, with the structure of 8-13-1 to predict the freight pricing of the order and test the prediction results. The test shows that the goodness of fit $\left(R^{2}\right)$ of the predicted value is close to 1 , and the prediction error range is less than $3.7 \%$, which proves the accuracy and effectiveness of the BP neural network model and provides an effective reference for the optimization of the pricing model of the car-free carrier platform.
\end{abstract}

\section{Introduction}

Road freight is a key part of comprehensive transportation construction and the main carrier for the rapid development of the modern logistics industry. However, while the road transportation continues to open up, many drawbacks have appeared, and the development status of "small, scattered, and chaotic" has gradually formed, and the development of the transportation industry has entered a bottleneck stage. Under the development environment of "internet + logistics," the "car-free carrier" model in the freight market has emerged, and car-free carrier companies have flourished.

The car-free carrier platform is a platform-type road freight intermediary who signs a transportation contract with the shipper as the carrier, assumes the responsibilities and obligations of the carrier, and then entrusts the actual carrier to complete the transportation task. This type of platform has the advantages of strong resource integration ability, wide brand effect, and convenient network promotion. Car-free carrier platforms need to intervene in freight transactions and earn freight difference. Compared with the direct transaction between the shipper and the carrier, the car-free carrier builds an information platform to promote information communication between the two parties, implements management model innovation, integrates and reconfigures bilateral channels, provides customers with standardized services, and improves transportation efficiency. Reduced transportation costs standardized the operation of the road freight industry. Countries with great transportation needs such as China provide opportunities for the development of car-free carrier platforms. Therefore, how to scientifically price the freight tasks of the car-free carrier platforms has strong research value and practical significance [1-5].

However, due to short development time and unbalanced resource allocation, the pricing mechanism of most car-free carrier platforms is imperfect, or even has major defects, and lacks strong theoretical support. How to better improve the pricing mechanism of the carrier business has become a major obstacle currently restricting the development of this industry, and an effective solution is urgently needed. Therefore, we take China as an example to study the 
status quo of the development of car-free carrier platforms and for in-depth analysis of the main factors that affect the pricing mechanism of the platform. And on the basis of the current mainstream pricing model, we propose to establish a transportation pricing optimization algorithm based on machine learning.

\section{Current Research Status}

The pricing strategy and mode of transportation routes are of vital importance to the transportation industry market, and they are also an important component of marketing. Georg and Stefan inspected transportation prices through three labor supply models and studied the impact of changes in the optimal level of transportation taxes on the above models [6]. Harald and Bjørn constructed an improved freight revenue assessment framework based on the number of economic orders and detailed transportation costs, combined with the limitations of the size and capacity of transportation vehicles [7]. Pavón and Rizzi took Santiago, Chile, as an example, assuming the relevant parameters. A simulation analysis of the interaction between the city's transportation price, transportation service provision, and cost recovery was performed [8]. Xuan and Lee established the Stackelberg model in the process of studying the transportation pricing problem with discount in the land port system composed of a land port corresponding to multiple shippers, obtained the global optimal solution by using a single breakpoint discount pricing scheme, and finally tested the effectiveness of quantity discount pricing in various situations [9]. Luis et al. took the Madrid metropolitan area of Spain as the research object and established a new optimization algorithm to optimize the four types of transportation policies and deeply explore the potential through the establishment of a land-use model and a transportation interaction model [10]. Based on marginal cost pricing rules, by simulating the user interaction process at the microlevel and introducing external factors such as the prolonged waiting time caused by passenger behavior, a pricing model of the best bus fare is constructed [11]. On the basis of game theory, Feng and Shi conducted an in-depth study on the transportation price and service quality of railway and freight transportation and then established the Hotelling model [12]. With the increasing development of China's transportation industry, car-free carrier platforms have begun to emerge in recent years. Since the development of car-free carrier platforms in China is still in the exploratory stage, there are relatively few documents available for reference. Xiong and Su established a multiple linear regression model and an improved GA-BP model, then built a neural network decision tree prediction model based on the first line pricing and related data, and achieved the maximum rationalization of pricing by adjusting the value of information entropy [13]. In addition, other documents mainly use game models, linear regression, fitting, and other traditional mathematical model methods to study the pricing of car-free carrier platforms. For example, Nie and Li used asymmetric information theory to construct a pricing model from the perspective of game [14].
Also, most of these documents only put forward relevant suggestions on the optimization of the platform's pricing mechanism from the policy level. Due to the limitations of traditional mathematical models, fewer factors are considered when constructing the pricing model of the platform. At the same time, it lacks the support of valid data and fails to achieve quantitative analysis. Therefore, the current research in this area generally has strong subjectivity. A scientific and reasonable route pricing model is crucial to the operating performance of the car-free carrier platform and the operational efficiency of the freight market. Therefore, the establishment of a comprehensive and perfect cargo consignment pricing model is of great significance and value to Chinese society.

\section{Research Ideas}

Based on the collected historical transaction data of a carfree carrier platform, this article has solved the pricing problem for actual carrier users from the perspective of carfree carriers and establishes an optimization algorithm for platform pricing. This paper has used the gray correlation model and the $K$-means clustering model to explore the main factors that affect the pricing of the platform and built the BP neural network model with multilayer feedforward on this basis. With the help of error backpropagation thinking, the optimal pricing decision for the platform's orders is made through machine learning in order to promote the rapid transaction between the two sides of the platform, reduce the time cost of the transaction, greatly improve the operating efficiency of the platform, and maximize profits.

\section{Data Selection and Processing}

This article selects 15,498 historical successful transaction order data from a certain car-free carrier platform, and the data come from https://www.bestfriend.com. Each order includes 20 characteristic indicators, as shown in Table 1.

After entering the data, we found that some of the data are abnormal. Therefore, we preprocess the data in the following ways: (i) eliminate the abnormal order data which have exactly the same value of each characteristic indicator or too many missing values. (ii) For the order data with individual missing values, Lagrange interpolation is used to complete the missing values. (iii) Unify the format of all time data. (iv) Use the one-hot method to perform vector encoding processing on all discrete data such as $x_{7}$ and $x_{10}$, and normalize all continuous data.

At the same time, in order to ensure the rigor of the research, we also need to make the following assumptions to eliminate the interference of other factors on the research results: (i) at the current stage, the car-free carrier platform aims to accelerate transactions and lower carrier costs. (ii) Every task of the platform will be carried out. (iii) The route tasks are all fixed vehicle tasks; that is, a task needs to be completed by one vehicle of a certain type of vehicle, regardless of the load sharing task. 
TABLE 1: The information of 20 characteristic indicators.

\begin{tabular}{lc}
\hline Indicator & Definition \\
\hline$x_{1}$ & Total mileage of the route \\
$x_{2}$ & Vehicle tonnage \\
$x_{3}$ & Vehicle length \\
$x_{4}$ & Transport class \\
$x_{5}$ & Planned time \\
$x_{6}$ & Actual time spent \\
$x_{7}$ & Area type \\
$x_{8}$ & Price adjustment ratio \\
$x_{9}$ & Transaction time \\
$x_{10}$ & Destination outlet \\
$x_{11}$ & Originating outlet \\
$x_{12}$ & Line coding \\
$x_{13}$ & Time allocated \\
$x_{14}$ & Adjusted time \\
$x_{15}$ & Whether to renew \\
$x_{16}$ & Packing type \\
$x_{17}$ & Business type \\
$x_{18}$ & Types of price adjustment \\
$x_{19}$ & Urgency of demand \\
$x_{20}$ & Type of requirement \\
\hline
\end{tabular}

\section{Model Establishment and Empirical Analysis}

5.1. Mining the Main Factors That Affect the Pricing of the Platform-Based on the Gray Relational Model. The model of gray correlation, that is, the degree of correlation between factors is measured according to the degree of similarity or difference in the development of many factors. It reveals the characteristics and degree of dynamic correlation of things. Analyzing the development and change of a system provides a more accurately quantitative measure. The basic principle of the model is to conduct an association analysis on the similarity between the independent variable and the dependent variable. The higher the similarity between the two curve shapes, the higher the correlation between the two. Conversely, the lower the similarity between the two, the lower the degree of association; otherwise, the correlation is lower [15-19]. The algorithm process is as follows.

Let $X_{i}(i=1,2,3, \ldots \ldots, 20)$ be the 20 characteristic indicators in the data we selected, and $X_{0}=\left(x_{0}(1)\right.$, $\left.x_{0}(2), \ldots, x_{0}(n)\right)$ are the corresponding orders' pricing. Make $\gamma\left(X_{0}, X_{i}\right)$ to be the gray correlation degree between $X_{0}$ and $X_{i}$. The expression is shown as follows:

$$
\begin{aligned}
& \gamma\left(X_{0}, X_{i}\right)=\frac{1}{n} \sum_{k=1}^{n} \gamma\left(x_{0}(k), x_{i}(k)\right), \\
& \gamma\left(x_{0}(k), x_{i}(k)\right) \\
& \quad=\frac{\min _{i} \min _{k}\left|x_{0}(k)-x_{i}(k)\right|+\xi \max _{i} \max _{k}\left|x_{0}(k)-x_{i}(k)\right|}{\left|x_{0}(k)-x_{i}(k)\right|+\xi \max _{i} \max _{k}\left|x_{0}(k)-x_{i}(k)\right|} .
\end{aligned}
$$

Among formulas (1) and (2), $\xi$ is the resolution coefficient, and the value range is $(0,1)$.

The calculation steps of the gray correlation degree are as follows:
Step 1: initialize the sequence data:

$$
x_{i}^{\prime}(k)=\frac{x_{i}(k)}{x_{i}(1)}, \quad i=0,1, \ldots, m, k=1,2, \ldots, n .
$$

Step 2: calculate the difference of the dependent variable influencing the factor sequence:

$$
\Delta_{i}=\left(\Delta_{i}(1), \Delta_{i}(2), \ldots, \Delta_{i}(n)\right), \quad i=1,2, \ldots, m .
$$

$$
\Delta_{i}(k)=\left|x_{0}^{\prime}(k)-x_{i}^{\prime}(k)\right|
$$

Step 3: calculate the maximum value $M$ and the minimum value $m$ in $\Delta_{i}(k)$ :

$$
\begin{gathered}
M=\max _{i} \max _{k} \Delta_{i}(k), \\
m=\min _{i} \min _{k} \Delta_{i}(k) .
\end{gathered}
$$

Step 4: calculate the correlation coefficient as follows:

$$
\gamma\left(x_{0}(k), x_{i}(k)\right)=\frac{m+\xi M}{\Delta_{I}(k)+\xi M} .
$$

Step 5: calculate the gray correlation degree:

$$
\gamma\left(X_{0}, X_{I}\right)=\frac{1}{n} \sum_{k=1}^{n} \gamma\left(x_{0}(k), x_{i}(k)\right) \text {. }
$$

Through formulas (3) to (9), we have obtained the gray correlation degree of each characteristic indicator and platform line pricing and sorted it, as shown in Table 2. At the same time, we use the $K$-means clustering model to cluster 20 feature indicators [20-24]. According to the AIC and $\mathrm{BIC}$, the 20 feature indicators are clustered into four categories, as shown in Figure 1.

According to the calculated gray correlation degree between each characteristic indicator and platform pricing and the $K$ means clustering result, from Table 2and Figure 1, we can see that the total mileage of the route $\left(x_{1}\right)$, planned time $\left(x_{5}\right)$, vehicle tonnage $\left(x_{2}\right)$, vehicle length $\left(x_{3}\right)$, and urgency of demand $\left(x_{19}\right)$ all have gray correlation coefficients higher than 0.75 with platform pricing and are grouped into one category, indicating that these five indicators are significantly related to platform pricing, which affects the platform line pricing major factor, but the gray relational algorithm cannot deeply analyze the inherent nonlinear relationship between variables. Therefore, based on the above conclusions, with the help of machine learning algorithms, we have deeply explored the internal connection between various indicators and platform transportation pricing.

5.2. Optimal Model of Transportation Pricing Based on BP Neural Network Algorithm. In order to maximize the rationalization of transportation pricing, the platform needs to work out an order price that can best match the final transaction price in the shortest possible time. While satisfying the interests of both parties on the platform, it greatly 
TABLE 2: The calculation results of the degree of gray correlation.

\begin{tabular}{lcc}
\hline Ranking & Indicator & Degree of relevance \\
\hline 1 & Total mileage of the route & 0.98274 \\
2 & Planned time & 0.94328 \\
3 & Vehicle tonnage & 0.83179 \\
4 & Urgency of demand & 0.82017 \\
5 & Vehicle length & 0.79424 \\
6 & Transport level & 0.63961 \\
7 & Destination outlets & 0.58013 \\
8 & Price adjustment ratio & 0.54481 \\
9 & Packing type & 0.40112 \\
10 & Originating outlet & 0.22149 \\
11 & Adjusted time & 0.20163 \\
12 & Line code & 0.11295 \\
13 & Business type & 0.06021 \\
14 & Type of requirement & 0.05916 \\
15 & Actul time & 0.05391 \\
16 & Type of price adjustment & 0.05128 \\
17 & Time allocated & 0.02012 \\
18 & Area type & 0.01232 \\
19 & Transaction time & 0.00412 \\
20 & Whether to renew & 0.00011 \\
\hline
\end{tabular}

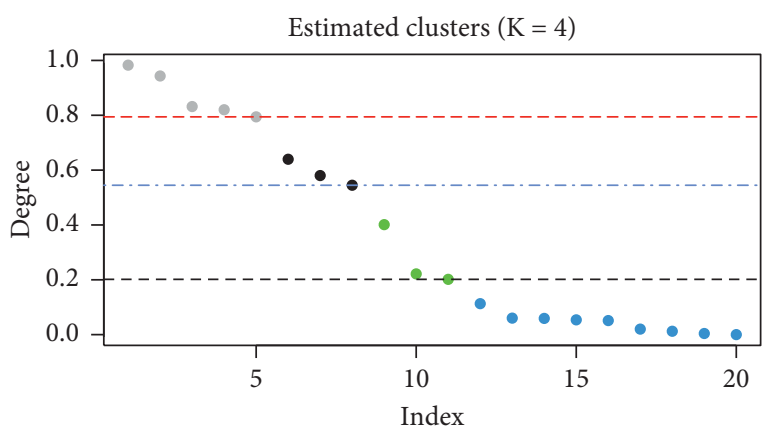

FIgURE 1: The graph of the $K$-means clustering result.

reduces the time and transportation costs spent in the quotation process. The BP neural network algorithm is a machine learning algorithm with integrated learning based on the principle of error backpropagation. We use the back propagation algorithm to adjust the weight and thresholds through the training error to make it reach the best state. Through the training of training samples, this can effectively reduce the output error caused by the algorithm and to realize the efficient learning of nonlinear relations in the sample data [25-28]. Therefore, we built a multilayer feedforward neural network model based on the BP algorithm to achieve machine deep learning of the final transaction price in the order data and built a pricing optimization algorithm that maximizes the platform's pricing. Finally, the model's pricing forecast results are tested to prove the rationality of the pricing model.

5.2.1. Model Algorithm and Principle. The structure of the BP neural network is divided into input layer, hidden layer, and output layer. The input layer and output layer are both one-layer structure, but the hidden layer can be one or more
[29-35]. In this article, we constructed a model with 3 hidden layers. Under normal circumstances, the relationship between the number of hidden layer networks $\left(n_{1}\right)$ and the number of input layer neurons $\left(n_{2}\right)$ is shown as follows:

$$
n_{1}=2 * n_{2}+1
$$

The structure of the BP neural network and the neuron model are shown in Figures 2and 3.

At the same time, based on the number of training sample sets, the hidden layers, weights, and the thresholds can be precisely calculated. According to Table 2and Figure 1, we believe that, among the 20 characteristic indicators, the 8 characteristic indicators clustered into the first two categories will have a significant impact on the pricing of the platform. Since other indicators are less relevant to pricing, we do not consider them. Therefore, the independent variable dimension of the neural network constructed in this article is 8 , so the number of neurons in the input layer is 8 . The dependent variable is a one-dimensional vector, which is the final transaction price of the order. Therefore, this paper constructs a network structure of 8-17-1.

Then, we trained the neural network, setting the abort error condition and normalizing the data in the training set. After this, inputting it into the neural network. Secondly, the neuron transfer functions of the hidden layer and the output layer are constructed using the tangent function of the output range $[-1,1]$, and the LM method was chosen as the training algorithm of the $\mathrm{BP}$ network; the neural network training is completed [36-41]. The specific training process is as follows:

Step 1: setup initialization:

We need to set the maximum number of training times, error conditions, initial weights, and thresholds of the neural network. Different initial weights and threshold settings will have a significant impact on the training effect of the network.

Step 2: sample training:

(1) Input training sample set $\left(X_{q}, T_{q}\right)$, where $q=1,2,3$, ....., $Q$

(2) For each input sample, calculate the output value of each neuron in sequence as follows: $g_{j}^{p}=f\left(\sum_{i=0}^{n 1} w_{j l} x_{p}^{l}\right), h_{k}^{p}=f\left(\sum_{j=0}^{n 2} w_{k j} g_{j}^{p}\right)$, and $y_{l}^{p}=$ $f\left(\sum_{k=0}^{n 3} w_{l k} h_{k}^{p}\right)$.

Step 3: error backpropagation:

Calculate the equivalent error of each layer of neurons, from the output layer to the input layer, return to Step 2 after obtaining the equivalent error, and perform forward propagation calculation and error backpropagation for other training sample pairs until all the training samples have gone through the above calculation steps.

Step 4: adjust the connection weight of each layer:

For the connection weight $w_{i j}$ from the hidden layer to the output layer, the correction amount for each step is $\Delta w_{i j}$, which satisfies the following relationship: 


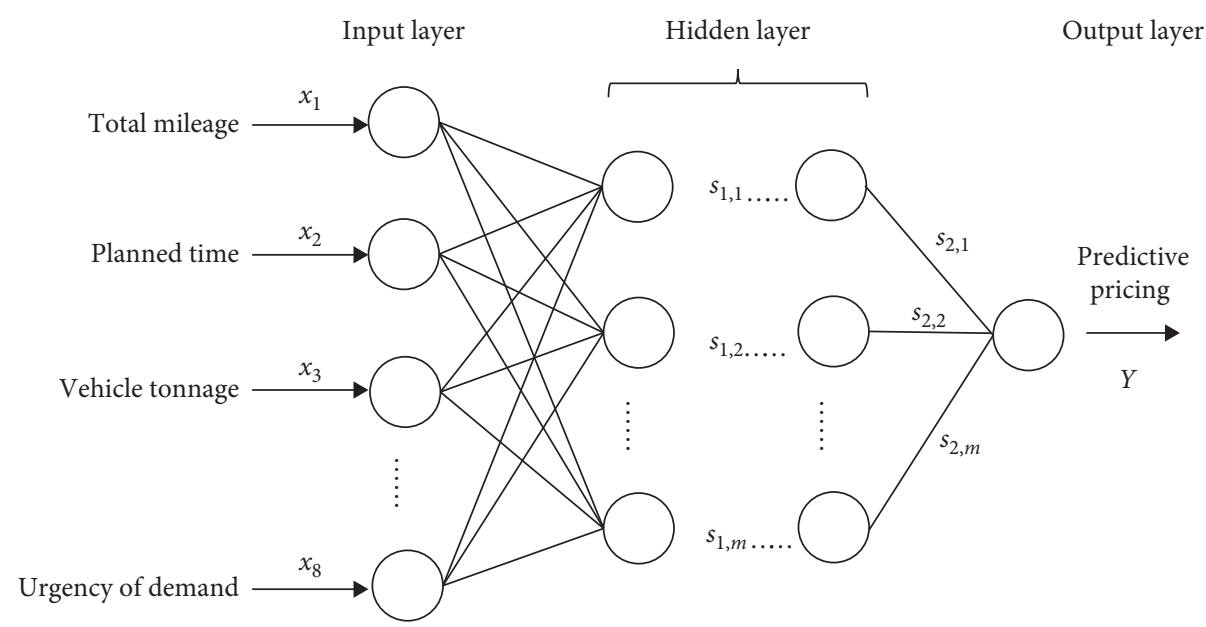

FIgURE 2: The diagram of the BP neural network structure.

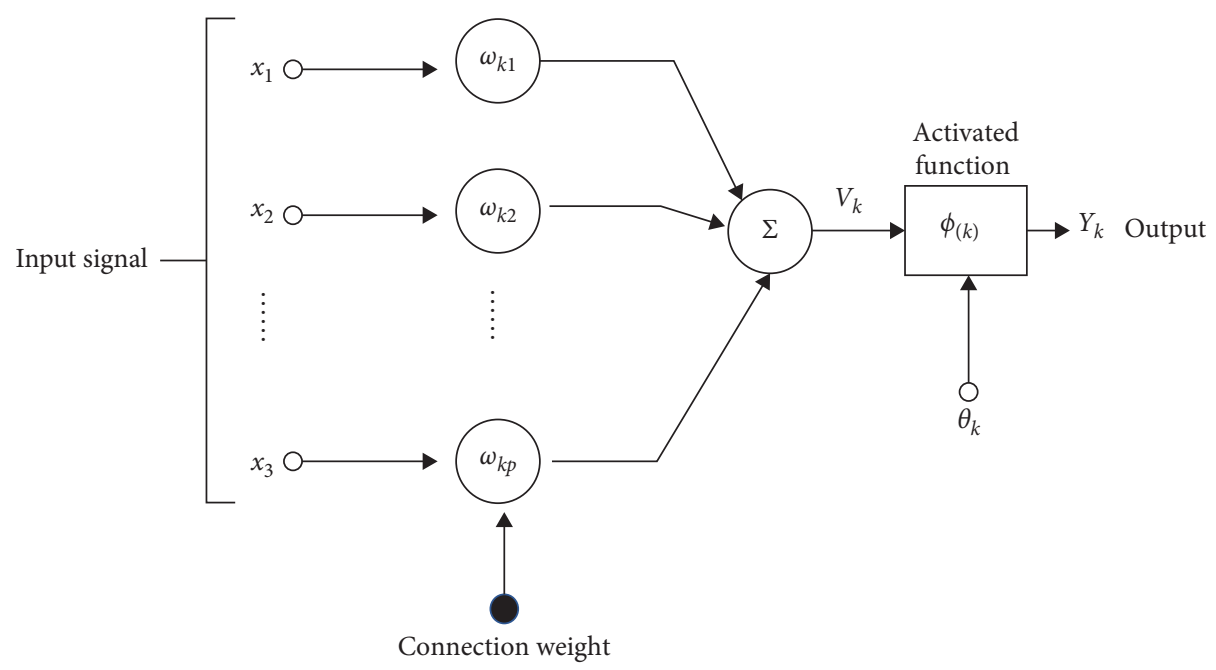

FIgURE 3: The structure of the neuron model.

$$
\begin{aligned}
\Delta w_{i j} & =-\eta \frac{\partial E}{\partial w_{i j}}=\eta \sum_{s}\left[T_{i}^{s}-O_{i}^{s}\right] \varphi^{\prime}\left(h_{i}^{s}\right) H_{j}^{s}=\eta \sum_{s} \delta_{i}^{s} H_{j}^{s}, \\
\delta_{i}^{s} & =\varphi^{\prime}\left(h_{i}^{s}\right)\left[T_{i}^{s}-O_{i}^{s}\right] .
\end{aligned}
$$

According to formulas (10) and (12), for the weight $\left(\bar{w}_{i j}\right)$ from the input layer to the hidden layer, the correction amount for each step is $\Delta \bar{w}_{i j}$, shown as follows:

$$
\begin{aligned}
\Delta \bar{w}_{i j} & =-\eta \frac{\partial E}{\partial w_{j k}}=\eta \sum_{s, j}\left[T_{i}^{s}-O_{i}^{s}\right] \varphi^{\prime}\left(h_{i}^{s}\right) w_{i j} \varphi^{\prime}\left(h_{i}^{s}\right) I_{j}^{s} \\
& =\eta \sum_{s, i} \delta_{i}^{s} w_{i j} \varphi^{\prime}\left(h_{j}^{s}\right) I_{k}^{s}=\eta \sum_{s} \bar{\delta}_{i}^{s} I_{k}^{s} .
\end{aligned}
$$

At this time, $\overline{\delta_{j}^{s}}$ satisfies the following formula:

$$
\bar{\delta}_{j}^{s}=\varphi \prime\left(h_{j}^{s}\right) \sum_{i} w_{i j} \delta_{i}^{s}
$$

Through formulas (13) to (15), the correction amount $\left(\Delta w_{p q}\right)$ of the connection weight between each layer in the neural network can be obtained, as shown in the following formula:

$$
\Delta w_{p q}=\eta \sum_{s} \delta_{p}^{s} v_{q}^{s}
$$

Step 5: forward calculation:

Return to Step 2, and then perform forward calculations based on the weights and thresholds calculated above. If the accuracy requirements are met, that is, for each training sample, its output layer neurons all meet the following accuracy requirement:

$$
\left|t_{l}^{p}-y_{l}^{p}<\varepsilon\right| \text {. }
$$



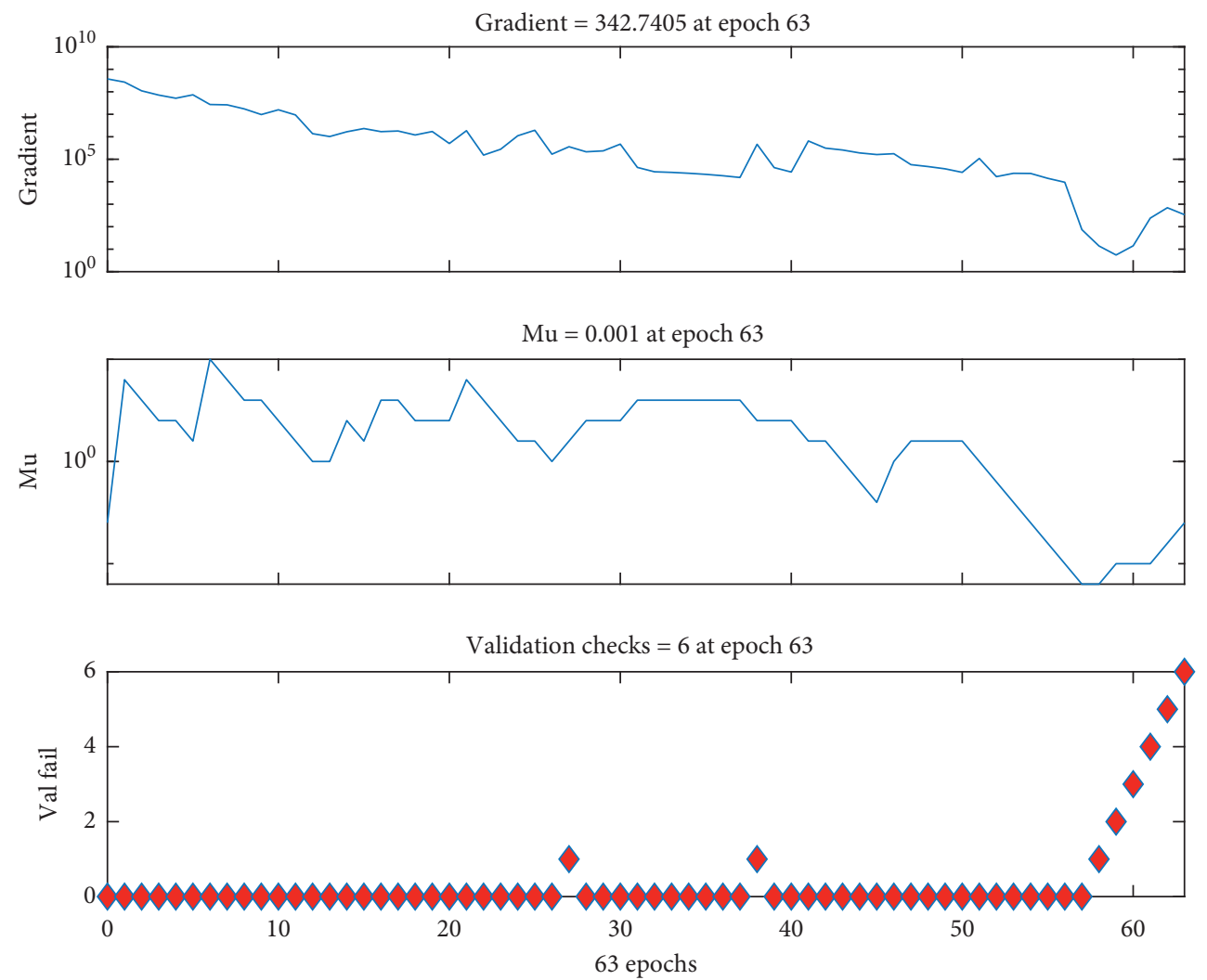

Figure 4: Data training process.

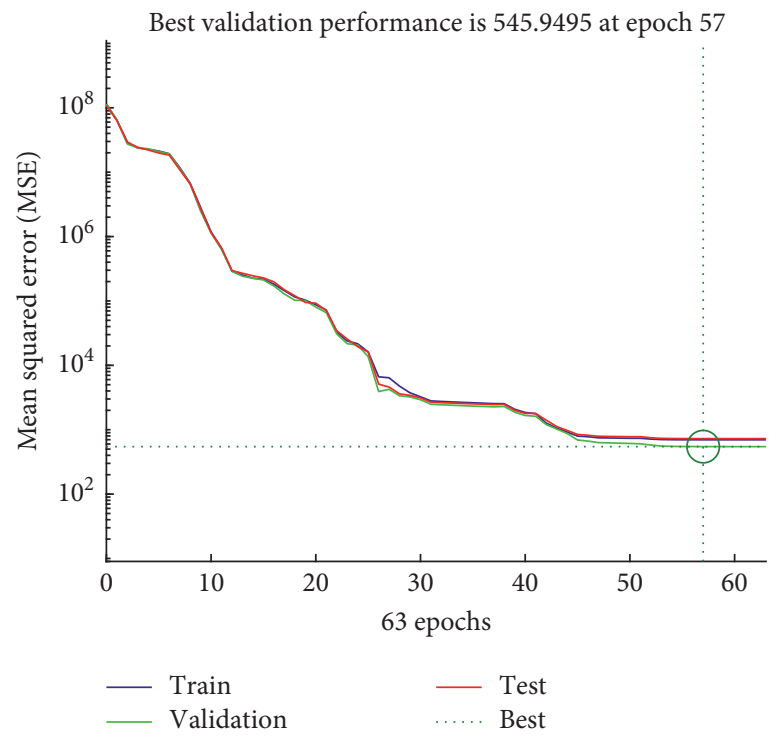

FIgURE 5: Data testing and iterative process.

Among formula (17), $p=1,2, \ldots \ldots, a$ and $l=1,2$, $\ldots \ldots, m$, where $\varepsilon$ is the given accuracy requirement value; when the accuracy requirement is met, the neural network training is completed; otherwise, it continues to iterate until the requirement is met.

Step 6: test the results:
Substitute the normalized test set into the trained BP neural network, and finally, get the test results.

5.2.2. Empirical Analysis of the Model. After testing, the optimal neural network structure selected for this question is 8-13-1. The input layer has 8 nodes, representing 8 

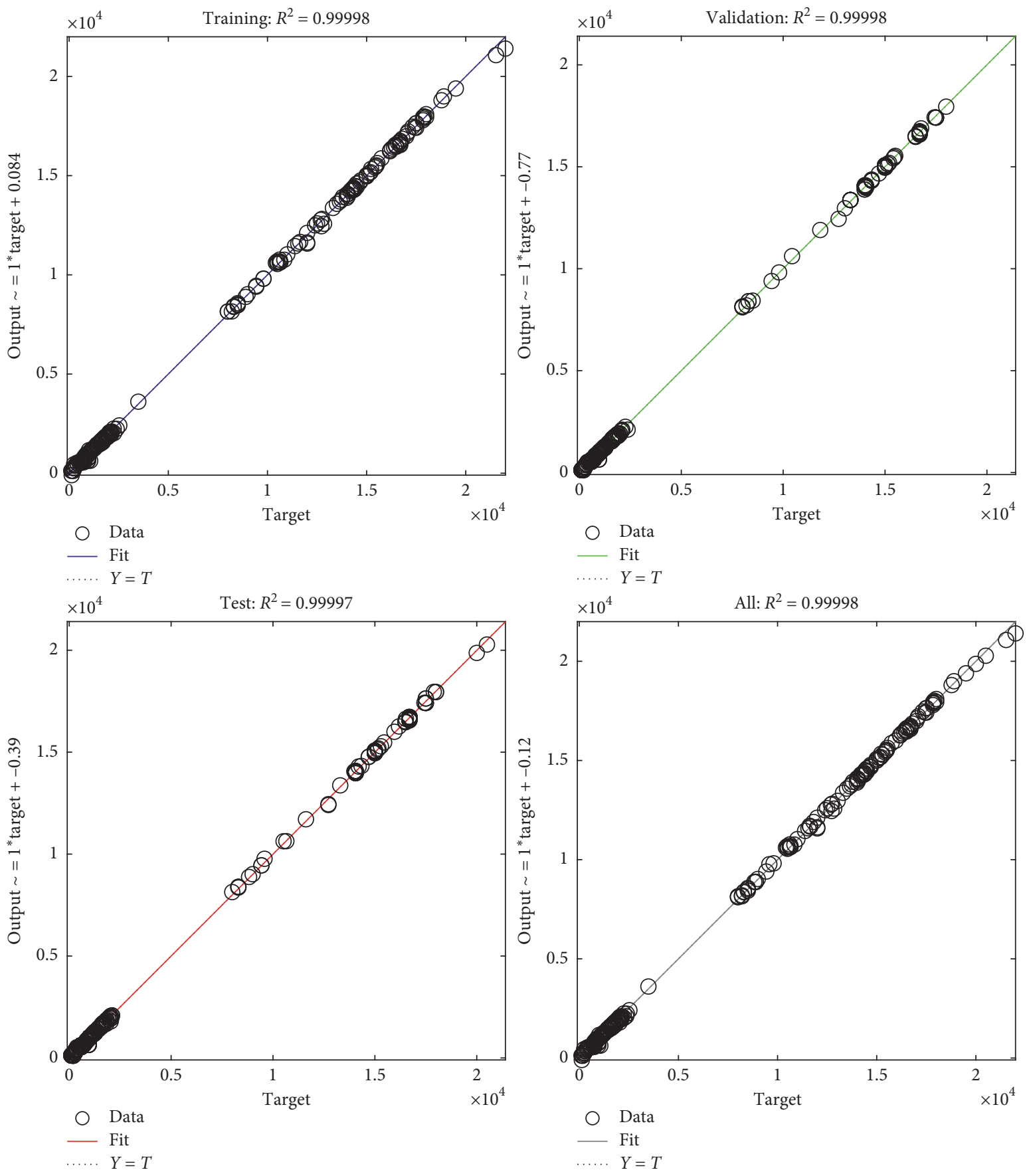

Figure 6: The test of regression fit.

characteristic indicators that affect transportation pricing, the hidden layer has 13 nodes, and the output layer has 1 node, which is the value for forecast pricing.

We use the collected 15,498 order data as samples and divide the training set, test set, and validation set according to the ratio of $70 \%, 15 \%$, and $15 \%$. Among them, the number of training sets is 11,211 , the number of validation sets is 2402 , and the number of test sets is 2403 .

After repeated calculations, we set the learning rate of the model to 0.01 , the learning momentum constant to 0.001 , and the maximum number of training times to 1000 . Then, we trained the training set data, and the process is shown in Figure 4.

Then, based on the training results of the above data, we selected the Levenberg-Marquardt algorithm to establish the BP neural network transportation pricing model and performed gradient descent on the data. The iterative test process is shown in Figure 5.

From Figure 5, we can see that when the test reaches the 57th time, the mean square error (MSE) reaches the minimum value, and the best model corresponds to the minimum MSE value. 


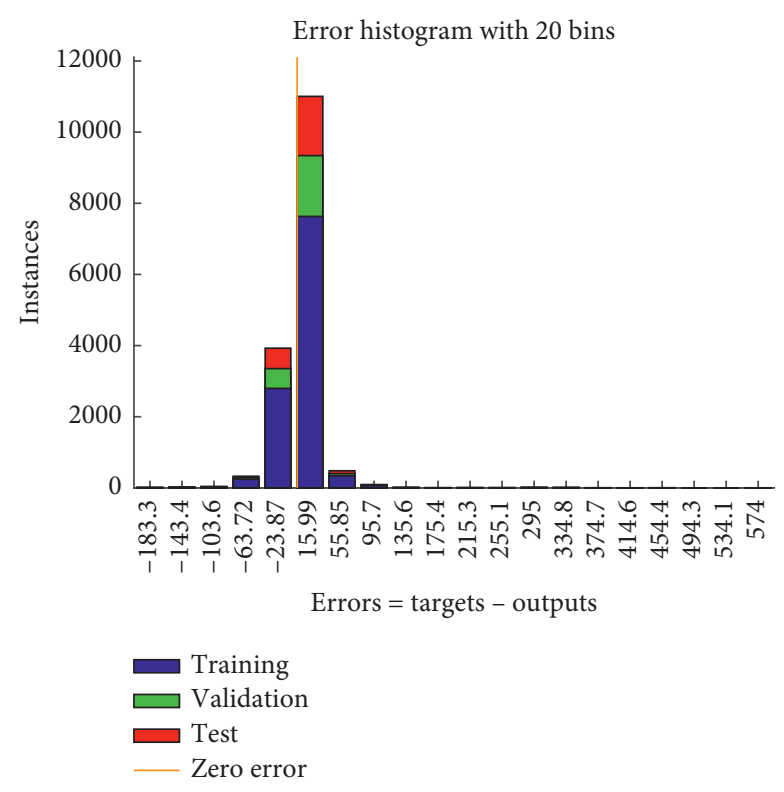

Figure 7: Error histogram.

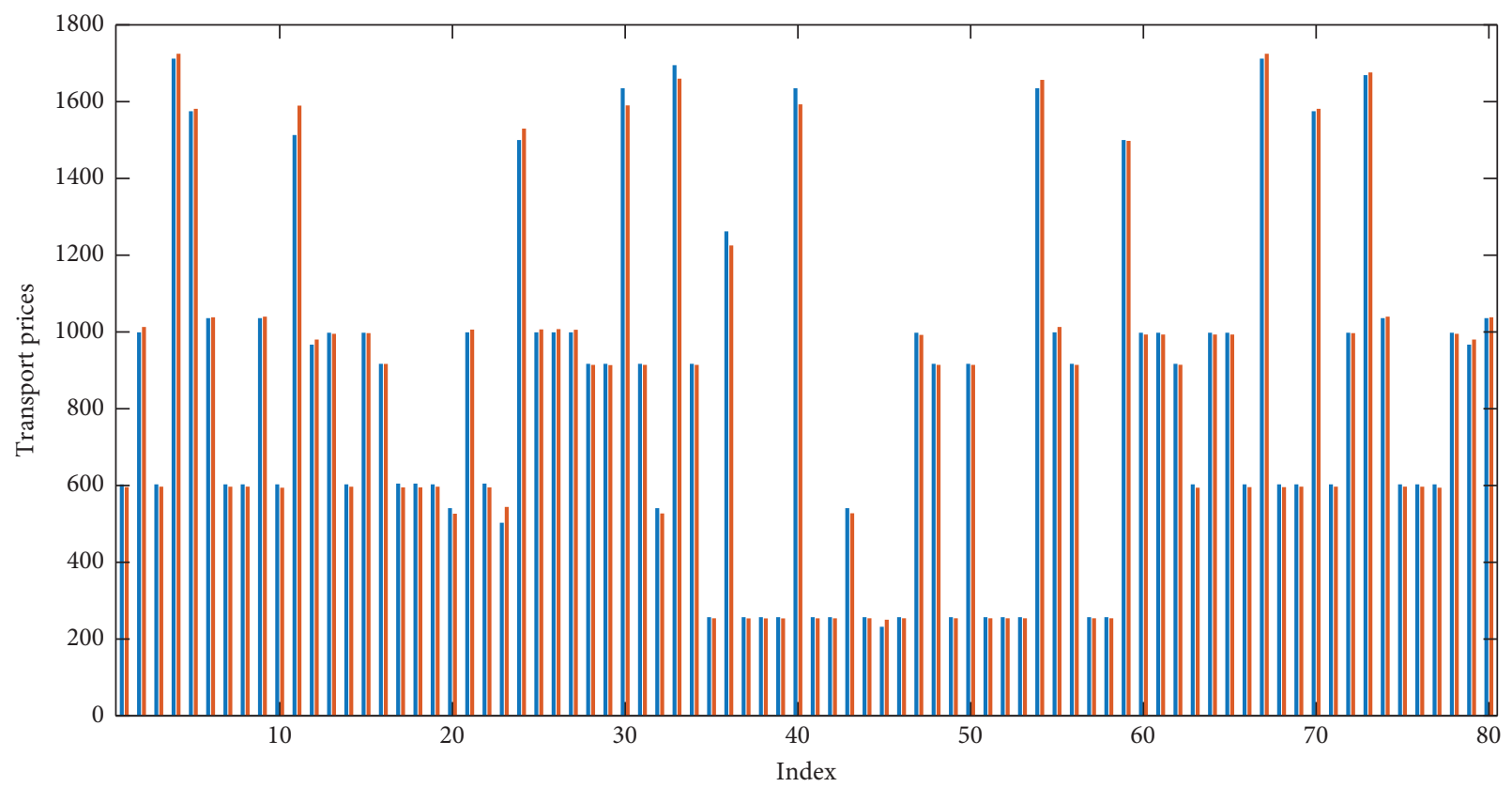

Figure 8: Comparison of line forecast price and actual price.

5.2.3. Validity Test of the Model. We have used the trained BP neural network model to predict transportation pricing and test the prediction results. We perform regression fitting on the predicted value of transportation pricing and test the goodness of fit $\left(R^{2}\right)$. The fitting result is shown in Figure 6 .

It can be seen from Figure 6that the goodness of fit $\left(R^{2}\right)$ between the predicted value of the test data and the measured value of transportation pricing is higher than 0.99 , and the effect of the model fitting is good. It shows that the established BP neural network has high stability, accuracy, and reliability. At the same time, according to the charts of the error histogram and the forecast comparison (Figures 7and 8), we can see that the predicted results of the model for transportation pricing are highly consistent with the actual transaction price of the order, which once again illustrates the accuracy and high applicability of the model, and we have realized the optimized design of the transportation pricing model. 


\section{Conclusion}

This paper took the car-free carrier platform in China as the research object, collected the historical order data, extracted relevant influencing factors, and built a platform route pricing optimization model based on the BP neural network algorithm. The main conclusions of this research are as follows.

By establishing a gray correlation model, we calculated the degree of correlation between each characteristic index in the historical transaction order and the final transaction price, established a $K$-means clustering model to cluster the above calculation results, and found "total mileage of the route," "vehicle tonnage," "vehicle length," "transport level," and "planned time" are the main factors that affect the pricing of the transportation route of the car-free carrier platform.

Based on the above results, we have established a BP neural network prediction model with the number of neurons in the input layer, hidden layer, and output layer being 8,13 , and 1 , respectively. Through the training of the training set data and the error backpropagation calculation, the neural network is tested, and the model is optimized when the number of iterations reaches the 57th time. Finally, we test the prediction results of the neural network algorithm according to the fitting coefficient $R^{2}$ and the prediction error value and found that the model fitting effect was good, and $R^{2}$ was close to 1 . And the error range between the predicted price and the actual transaction price is generally less than $3.7 \%$. It shows that the prediction accuracy of this model is high, stable, and reliable. The optimized forecast of platform transportation pricing is realized.

Based on the above research conclusions, we put forward the following three suggestions:

(1) Car-free carrier platforms should change the transportation pricing system based on traditional regression prediction models and use more accurate and time-efficient artificial neural network models for pricing

(2) When setting prices, the platform should pay more attention to the influence of factors such as transportation mileage, relevant parameters of transportation vehicles, and the urgency of transportation

(3) It is suggested that the noncar carrier platform can adopt the mobile quotation mechanism, and the transportation pricing can be dynamically adjusted with the change of time to increase the transaction rate of the platform

\section{Data Availability}

The data used to support the findings of this study are included within the article.

\section{Conflicts of Interest}

The author declares that there are no conflicts of interest regarding the publication of this paper.

\section{Acknowledgments}

This study was funded by the Humanities and Social Sciences Research Major Project of the Education Department of Anhui Province (SK2017A0452), the Teaching and Research Fund Project of the Education Department of Anhui Province (2020jyxm0017), "First-Class Course" of Anhui University of Finance and Economics (acylkc202008), and the Teaching and Research Fund Project of the Anhui University of Finance and Economics (acjyyb2020011 and acjyyb2020014).

\section{References}

[1] Y.-J. Zhao, X.-Y. Duan, and J.-L. Gao, "Platform research on car free carrier based on the "internet +"' IOP Conference Series: Earth and Environmental Science, vol. 186, no. 6, p. 5, Article ID 012042, 2018.

[2] J.-Y. Zhang, "Discussion on the development of car-free carriers in China's logistics industry," Open Journal of Business and Management, vol. 7, no. 2, pp. 828-836, 2019.

[3] N. Singh, R. Gholami, P. Agrawal et al., "Information technology/systems adoption in the public sector: evidence from the Illinois department of transportation," Journal of Global Information Management, vol. 29, no. 4, pp. 172-194, 2021.

[4] P.-H. Zeng and X.-C. Wei, "Measurement and convergence of transportation industry total factor energy efficiency in China," Alexandria Engineering Journal, vol. 60, no. 5, pp. 4267-4274, 2021.

[5] H.-R. Deng, "Research and analysis of transportation engineering planning and transfer traffic volume based on cube," Journal of Physics: Conference Series, vol. 1744, no. 2, p. 5, Article ID 022038, 2021.

[6] H. Georg and T. Stefan, "The role of labor-supply margins in shaping optimal transport taxes," Economics of Transportation, vol. 22, no. 6, p. 16, Article ID ID100156, 2020.

[7] M. Harald and G. J. Bjørn, "A logistics cost function with explicit transport costs," Economics of Transportation, vol. 19, no. 9, p. 13, Article ID 100116, 2020.

[8] N. Pavón and L. I. Rizzi, "Road infrastructure and public bus transport service provision under different funding schemes: a simulation analysis," Transportation Research Part A: Policy and Practice, vol. 125, no. 7, pp. 89-105, 2019.

[9] Q. Xuan and C.-Y. Lee, "Quantity discount pricing for rail transport in a dry port system," Transportation Research Part E: Logistics and Transportation Review, vol. 122, no. 2, pp. 563-580, 2019.

[10] A. G. Luis, D. L. H. Daniel, and M. Andrés, "Optimization of transport measures to reduce GHG and pollutant emissions through a LUTI modeling approach," International Journal of Sustainable Transportation, vol. 10, no. 7, pp. 590-603, 2016.

[11] K. Ihab, K. Benjamin, N. Andreas et al., "Agent-based optimisation of public transport supply and pricing: impacts of activity scheduling decisions and simulation randomness," Transportation, vol. 42, no. 6, pp. 1039-1061, 2015.

[12] F.-L. Feng and X. Shi, "Option pricing model by railway cargo transport based on Heston model and genetic algorithm," Journal of Railway Science and Engineering, vol. 17, no. 5, pp. 1295-1301, 2020.

[13] Z.-Y. Xiong and T.-K. Su, "Dynamic game pricing model of car-free carrier platform based on GA-BP theory," Journal of Physics: Conference Series, vol. 1629, no. 1, p. 9, Article ID 012053, 2020 
[14] F. H. Nie and D. S. Li, "Study of pricing strategies for non-car operating carriers platform under asymmetric information," Industrial Engineering and Management, vol. 25, no. 5, pp. 121-128, 2020.

[15] U. Dukpa Urgen and S. Basel, "Socio-economic development of Darjeeling Himalayas: categorical principal component analysis (CATPCA) and ordinal logistic regression (OLR)," Indian Journal of Economics and Development, vol. 17, no. 1, pp. 128-135, 2021.

[16] Y.-K. Huang, W.-D. Jin, Z.-B. Yu et al., "A robust anomaly detection algorithm based on principal component analysis," Intelligent Data Analysis, vol. 25, no. 2, pp. 249-263, 2021.

[17] Y. Kim, J. Lee, and T. D. Little, "Multiple imputation with principal components for non-normal categorical data," Multivariate Behavioral Research, vol. 56, no. 1, pp. 165-166, 2020.

[18] Y. Jindal, Y. Rajesh, and D. S. Phogat, "Principal component analysis and determination of the selection criteria in fodder cowpea (Vigna unguiculata (L) Walp.) genotypes," Range Management and Agroforestry, vol. 39, no. 2, pp. 191-196, 2018.

[19] A. Ali, B. M. Margetts, and A. A. Zainuddin, "Exploration of the principal component analysis (PCA) approach in synthesizing the diet quality of the Malaysian population," $\mathrm{Nu}$ trients, vol. 13, no. 1, pp. 70.1-70.21, 2020.

[20] M. H. Ghaffari, H. M. Hammon, D. Frieten et al., "Effects of milk replacer meal size on feed intake, growth performance, and blood metabolites and hormones of calves fed milk replacer with or without butyrate ad libitum: a cluster-analytic approach," Journal of Dairy Science, vol. 104, no. 4, pp. 4650-4664, 2021.

[21] S. Sahar, D. Hamed, A. H. B. Seyed et al., "Fully automatic 3D segmentation of the thoracolumbar spinal cord and the vertebral canal from T2-weighted MRI using K-means clustering algorithm," Spinal Cord, vol. 58, no. 7, pp. 811-820, 2020.

[22] J. Ashutosh Jha and S. Debashis, "Examining categorization of telecom circles in India using unsupervised $k$-means clustering on techno-economic indicators," Decision, vol. 46, no. 3, pp. 365-383, 2019.

[23] Y. Liu and B.-F. Li, "Bayesian hierarchical $K$-means clustering," Intelligent Data Analysis, vol. 24, no. 5, pp. 977-992, 2020.

[24] H. Sangheum and K. Dohyun, "A scalable feature based clustering algorithm for sequences with many distinct items," International Journal of Fuzzy Logic and Intelligent Systems, vol. 18, no. 4, pp. 316-325, 2018.

[25] F. Shriver, C. Gentry, and J. Watson, "Prediction of neutronics parameters within a two-dimensional reflective PWR assembly using deep learning," Nuclear Science and Engineering, vol. 195, no. 6, pp. 626-647, 2021.

[26] I. O. Khalaf and S. Dalal, "Prediction of occupation stress by implementing convolutional neural network techniques," Journal of Cases on Information Technology, vol. 23, no. 3, pp. 27-42, 2021.

[27] S. Eismann, R. J. L. Townshend, and N. Thomas, "Hierarchical, rotation-equivariant neural networks to select structural models of protein complexes," Proteins: Structure, Function, and Bioinformatics, vol. 89, no. 5, pp. 493-501, 2020.

[28] B. J. Kim, "Model selection in artificial neural network," International Journal of Advanced Smart Convergence, vol. 7, no. 4, pp. 57-65, 2018.

[29] W.-B. Yu and F.-Y. Zhao, "Prediction of critical properties of biodiesel fuels from FAMEs compositions using intelligent genetic algorithm-based back propagation neural network," Energy Sources, Part A: Recovery, Utilization, and Environmental Effects, vol. 43, no. 17, pp. 2063-2076, 2021.

[30] J.-C. Wang, B. Zou, and M.-F. Liu, "Milling force prediction model based on transfer learning and neural network," Journal of Intelligent Manufacturing, vol. 32, no. 4, pp. 947956, 2021.

[31] W.-P. Zhao, J.-C. Li, and J. Zhao, "Research on evaporation duct height prediction based on back propagation neural network," IET Microwaves, Antennas \& Propagation, vol. 14, no. 13, pp. 1547-1554, 2020.

[32] M. B. Jibrin, M. K. Aregbesola, and O. M. Agboola, "Diagnosis of diabetes mellitus type II using a feed forward back propagation neural network," Journal of Computer Science and Control Systems, vol. 13, no. 1, pp. 39-43, 2020.

[33] V. Geetha, K. S. Aprameya, and M. H. Dharam, "Dental caries diagnosis in digital radiographs using back-propagation neural network," Health Information Science and Systems, vol. 8, no. 3, pp. 1519-1532, 2020.

[34] K. A. Clement, A. T. Victor, and Y. Z. Yao, "Soft computingbased technique as a predictive tool to estimate blast-induced ground vibration," Journal of Sustainable Mining, vol. 18, no. 4, pp. 287-296, 2019.

[35] S. Norhazlina, A. G. Nurul, and Z. U. S. M. J. Ahmad, "Prediction of ozone concentration using feed forward back propagation neural network (FFBP-NN)," International Journal of Recent Technology and Engineering, vol. 8, no. 4, pp. 9257-9260, 2019.

[36] J.-X. Kang, Y.-J. Lu, H.-B. Luo et al., "Wear assessment model for cylinder liner of internal combustion engine under fuzzy uncertainty," Mechanics \& Industry, vol. 22, no. 29, p. 9, Article ID 2021028, 2021.

[37] J. B. Liu, J. Zhao, and J. Min, "On the Hosoya index of graphs formed by a fractal graph," Fractals-Complex Geometry Patterns and Scaling in Nature and Society, vol. 27, no. 8, pp. 19-35, 2019.

[38] X.-B. Liu, Y.-J. Zhang, W.-K. Cui et al., "Development assessment of higher education system based on TOPSIS-entropy, hopfield neural network, and cobweb model," Complexity, vol. 2021, Article ID 5520030, 11 pages, 2021.

[39] J.-M. Zhu, Y. Chen, and S. Zhang, "Analysis of the impact of climate change on national vulnerability based on fuzzy comprehensive evaluation," Discrete Dynamics in Nature and Society, vol. 2020, Article ID 3527540, 10 pages, 2020.

[40] F. Xu, Y.-A. Du, H. Chen et al., "Prediction of fish migration caused by ocean warming based on SARIMA model," Complexity, vol. 2021, Article ID 5553935, 9 pages, 2021.

[41] R. Xie, R. Liu, X.-B. Liu et al., "Evaluation of SMEs' credit decision based on support vector machine-logistics regression," Journal of Mathematics, vol. 2021, Article ID 5541436, 10 pages, 2021. 